\title{
Misoprostol-induced Coronary Vasospasm Complicated by Takotsubo Cardiomyopathy and Cardiac Arrest
}

\author{
Parminder Kaur ${ }^{1}$ Melvin Santana ${ }^{2}$, Balraj Singh ${ }^{3}{ }_{2}$ Anuraag Sah ${ }^{2}$, Raja Pullatt ${ }^{4}$, Fayez Shamoon ${ }^{1}$ \\ ${ }^{1}$ Department of Cardiology, Saint Joseph's University Medical Center, Paterson, NJ, USA \\ ${ }^{2}$ Department of Internal Medicine Trinitas Regional Medical Center, Elizabeth, NJ, USA \\ ${ }^{3}$ Department of Hematology-Oncology, Saint Joseph's University Medical Center, Paterson, NJ, USA \\ ${ }^{4}$ Department of Cardiology, Trinitas Regional Medical Center, Elizabeth, NJ, USA
}

\section{Doi: 10.12890/2021_002674 - European Journal of Case Reports in Internal Medicine - ๑ EFIM 2021}

Received: 29/05/2021

Accepted: 03/06/2021

Published: $23 / 06 / 2021$

\begin{abstract}
How to cite this article: Kaur P, Santana M, Singh B, Sah A, Pulllatt R, Shamoon F. Misoprostol-induced coronary vasospasm complicated by takotsubo cardiomiopathy and cardiac arrest. EJCRIM 2021;8: doi:10.12890/2021_002674.
\end{abstract}

Conflicts of Interests: The authors declare there are no competing interests.

This article is licensed under a Commons Attribution Non-Commercial 4.0 License

\section{ABSTRACT}

Misoprostol is a synthetic E1 prostaglandin commonly used to induce abortion in the United States and elsewhere. There is limited literature on the cardiovascular adverse effects of misoprostol, and, to the best of our knowledge, very few such events have been reported. We describe the case of 52-year-old woman who was given misoprostol for cervical softening before endometrial ablation and experienced a cardiac arrest due to coronary vasospasm. She was successfully resuscitated and echocardiography showed features consistent with Takotsubo cardiomyopathy. Coronary angiography revealed coronary artery spasm which responded to nitroglycerin. Our case adds to the limited literature on this life-threatening adverse event of misoprostol.

\section{LEARNING POINTS}

- Misoprostol is frequently used obstetrics and gynaecology and routinely for the medical termination of pregnancy.

- Misoprostol can result in cardiac arrest due to coronary vasospasm.

- Coronary vasospasm induced by misoprostol can be relieved with intra-arterial nitroglycerin.

\section{KEYWORDS}

Misoprostol, Takotsubo cardiomyopathy, coronary vasospasm

\section{INTRODUCTION}

Misoprostol is a synthetic prostaglandin E1 analogue that primarily effects the mucosa in the gastrointestinal tract, causing acid suppression. However, it also has off-label use in obstetrics and gynaecology owing to its ability to induce uterine contractions, cervical softening and dilation. Due to its uterotonic properties, it is used for the medical termination of pregnancy, postpartum haemorrhage and induction of labour ${ }^{[1]}$. Misoprostol is considered a safe and well-tolerated drug. We report the case of 52-year-old woman who was given misoprostol for cervical softening before endometrial ablation and had a cardiac arrest due to coronary vasospasm

\section{CASE DESCRIPTION}

We describe a 52-year-old Hispanic woman with a medical history of hypertension and dysfunctional uterine bleeding who was brought to the emergency department after she was found unresponsive. The patient had been given oral misoprostol $25 \mu \mathrm{g}$ for cervical ripening 24 
hours before she had undergone endometrial ablation for dysfunctional uterine bleeding at an outpatient facility. Initially she tolerated the misoprostol well, but after the procedure she developed nausea followed by chest pain and then became unresponsive. Cardiopulmonary resuscitation (CPR) was started by bystanders and the emergency medical services were called. The patient was noted to be in ventricular fibrillation (V-Fib); a shock was administered and normal sinus rhythm was restored. She was intubated in the field and transferred to the emergency room.

In the emergency room, the patient had an electrocardiogram (ECG) that showed sinus rhythm with first degree AV block, ST depression in the inferior and anterolateral leads, and left axis deviation (Fig. 1). Later, in the emergency room the patient again went into V-Fib and received three additional shocks. Cardiac rhythm was restored, but approximately 1 hour later the patient experienced another $\mathrm{V}$-Fib arrest and the return of spontaneous circulation was only achieved after 12 minutes of advanced cardiovascular life support. The patient was initiated on amiodarone infusion for the maintenance of sinus rhythm followed by transfer to the intensive care unit for further evaluation and management.

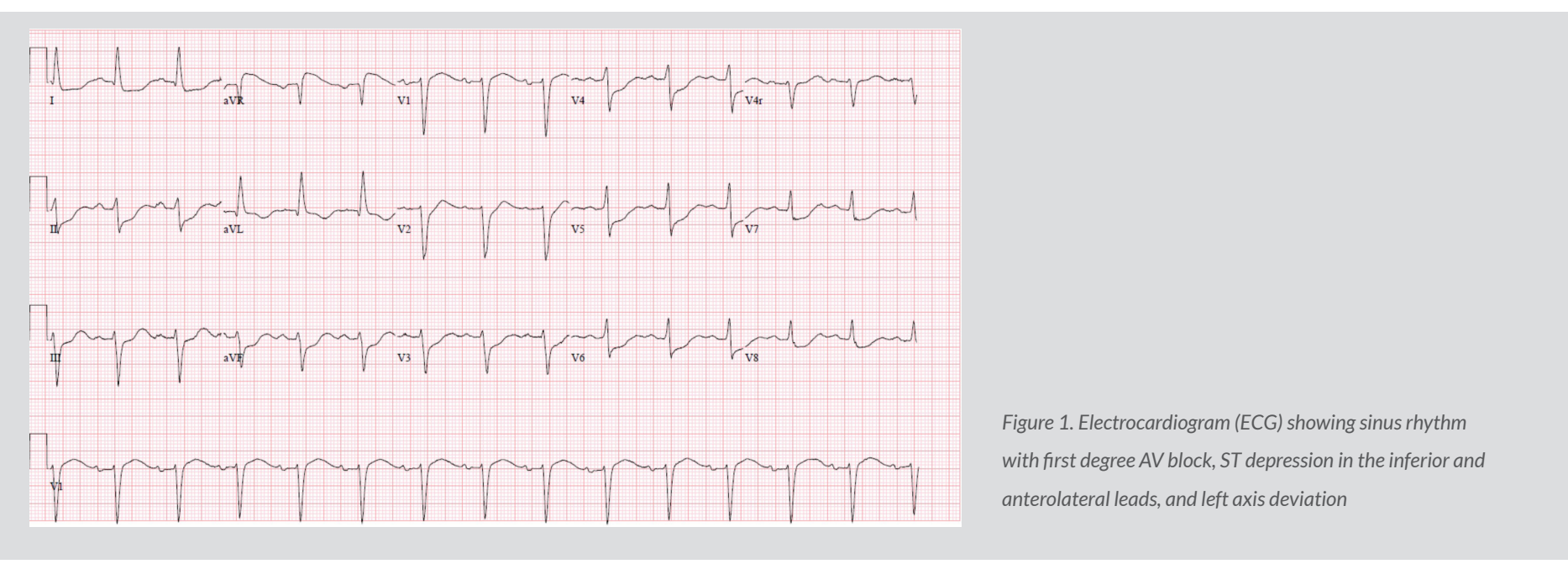

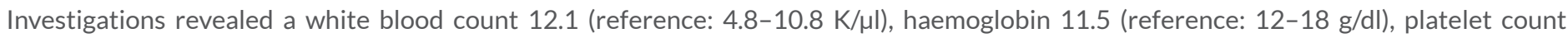
115 (reference: $150-400 \mathrm{~K}$ ), initial troponin 0.0 (normal <0.5 ng/ml), creatinine 0.93 (reference: 0.7-1.2 mg/dl), blood urea nitrogen 17 (reference: 8-20 mg/dl), sodium 138 (reference: 136-145 mmol/l), potassium 3.2 (reference: $3.6-5.1$ mmol/l), chloride 105 (reference: 101 $111 \mathrm{mmol} / \mathrm{l}$ ), bicarbonate 16 (reference: $22-28 \mathrm{mmol} / \mathrm{l}$ ), calcium 8.9 (reference: 8.9-10.3 mg/dl), glucose 247 (reference: $74-118 \mathrm{mg} / \mathrm{dl}$ ), aspartate aminotransferase 357 (reference: 15-41 U/I), alanine aminotransferase 319 (reference: 14-54 U/I), total bilirubin 1 (reference: 0.4-2 mg/dl), magnesium 2.1 (reference: 1.8-2.5 mg/dl), INR 1.1. A computed tomography angiogram of the chest showed no evidence of pulmonary embolism, while a transthoracic echocardiogram (TTE) revealed a left ventricle ejection fraction (EF) of 30-35\%, and severe hypokinesis of the apical and mid myocardial segments involving both the anterior and inferior walls and anterolateral and anteroseptal walls (Video 1). Subsequent cardiac catheterization showed coronary vasospasm in the left anterior descending artery (LAD) and insignificant $50 \%$ non-obstructive plaque in the proximal LAD (Fig. 2). Coronary vasospasm resolved after nitroglycerin was administered (Fig. 3). The patient was successfully extubated and discharged home. A repeat TTE performed 1 week later showed resolution of wall abnormalities and EF improved to $65 \%$.

\section{DISCUSSION}

Misoprostol is commonly used in obstetrics and gynaecology and routinely for the medical termination of pregnancy. Due to its predominant effect on the gastrointestinal mucosa, the most common side effects include diarrhoea, nausea, vomiting and abdominal cramping. It is also a teratogenic drug associated with several congenital foetal defects. Systemic infections have been rarely reported when misoprostol is used for medical abortion. However, serious adverse events have been described. Prashanth et al. report two cases of misoprostol-induced severe vasospasm leading to a hypertensive crisis requiring mechanical ventilation ${ }^{[2]}$. Illa et al. reported the case of a 32-year-old woman who developed an episode of transient acute coronary artery vasospasm following the administration of intravaginal misoprostol ${ }^{[3]}$. Owusu et al. described a case of successful cardiac resuscitation after the administration of intravenous nitroglycerin during prolonged cardiac arrest secondary to coronary artery vasospasm in the setting of recent misoprostol ingestion [4]. Mazhar et al. described the case of a 39-year-old woman with no major underlying cardiovascular risk factors who developed an episode of acute coronary syndrome following 


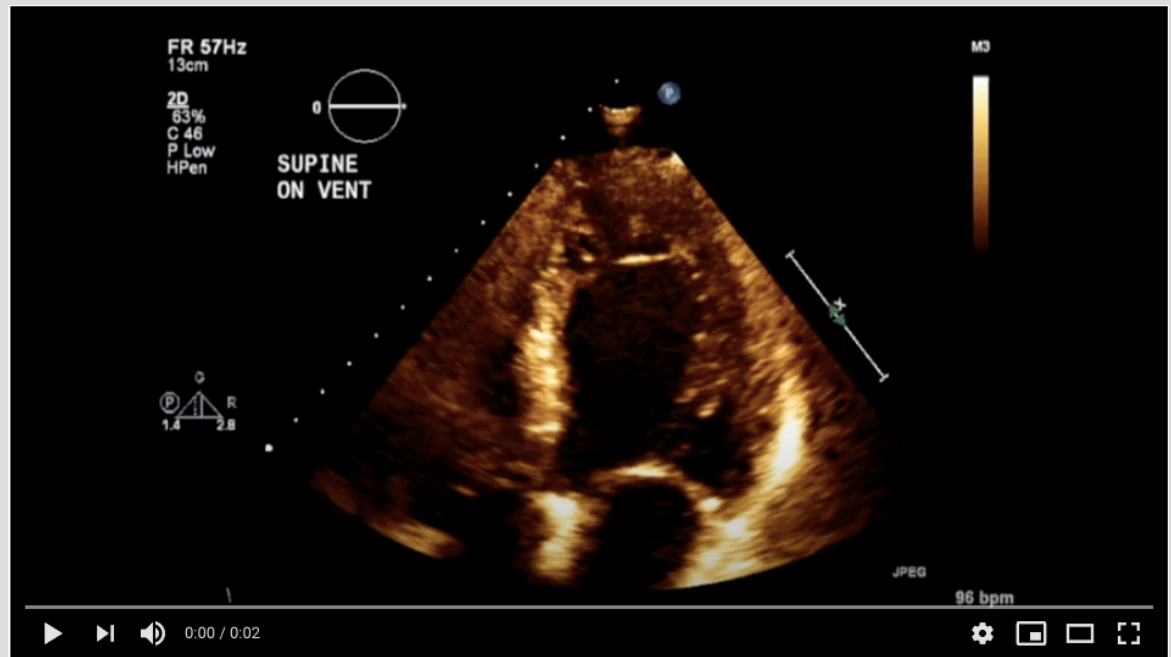

Video 1. Transthoracic echocardiogram (TTE) revealed a left ventricle ejection fraction (EF) of $30-35 \%$, and severe hypokinesis of the apical and mid myocardial segments involving both the anterior and inferior walls and anterolateral and anteroseptal walls

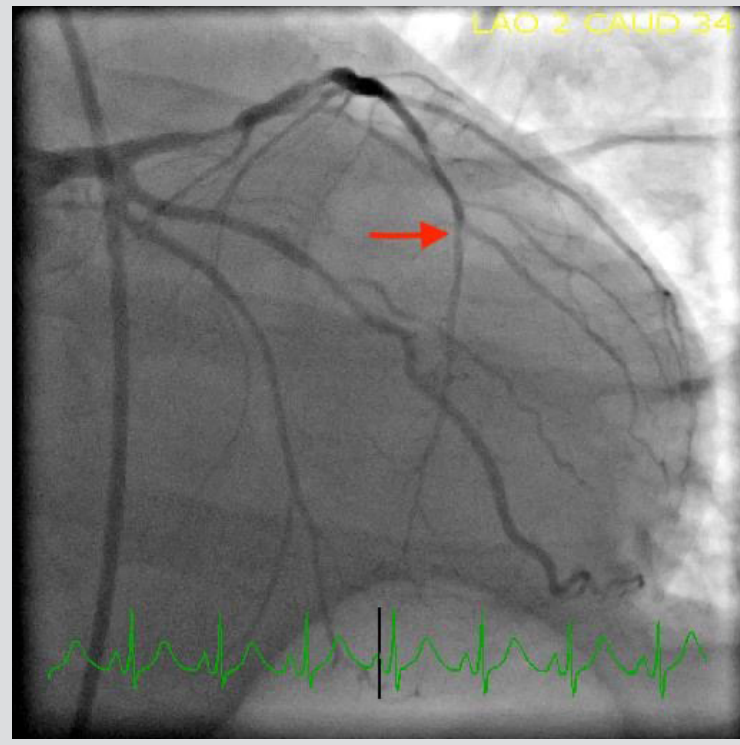

Figure 2. Cardiac catheterization showing coronary vasospasm in the left anterior descending artery (LAD) and 50\% non-obstructive plaque in the proximal LAD

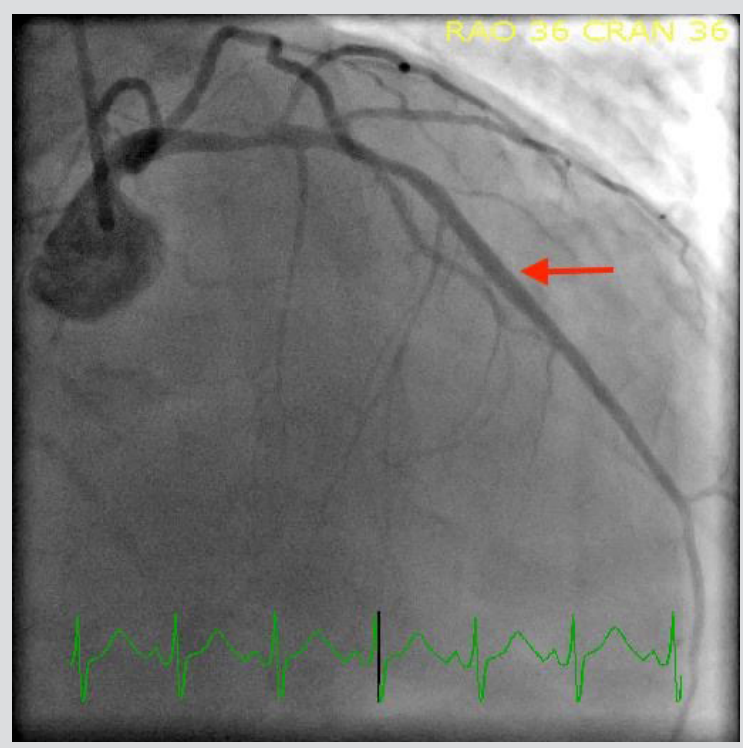

Figure 3. Cardiac catheterization showing the left anterior descending artery vasospasm resolved after nitroglycerin administration

the administration of two doses of misoprostol. However, the patient's symptoms completely resolved after misoprostol was discontinued ${ }^{[5]}$. The exact pathological mechanism leading to these adverse effects is not fully understood, but vasoconstriction appears to be the underlying feature due to activation of $G$ protein-coupled receptors, specifically subtypes EP1 and EP3 ${ }^{[6,7]}$.

However, unlike the above-mentioned cases, in addition to cardiac arrest following misoprostol and documented coronary vasospasm on coronary angiogram, our patient also had echocardiographic and ventriculogram findings consistent with Takotsubo cardiomyopathy (TTC), presumably secondary to coronary vasospasm. Several case reports have described TTC following coronary vasospasm. Brennan et al. describe a female patient who was found to have coronary vasospasm and TTC and suggest further study is needed into the association between these two conditions ${ }^{[8]}$. Misumi et al. reported a similar case of a 79-year-old man with right coronary artery spasm leading to TTC ${ }^{[9]}$. Another postulated mechanism for TTC is catecholamine excess but this is not supported by our case report, which highlights coronary artery vasospasm resulting from systemic misoprostol administration as the predominant mechanism. 


\section{CONCLUSION}

In conclusion, a 52-year-old woman was given misoprostol for cervical ripening 24 hours before endometrial ablation and experienced a sudden cardiac arrest due to coronary vasospasm accompanied by a depressed EF of 30-35\% and apical ballooning of the left ventricle consistent with TTC. Coronary vasospasm was subsequently relieved with intra-arterial nitroglycerin and a repeat TTE performed 1 week later showed normal EF. Our case and review of the literature adds to the limited literature on misoprostol-induced coronary vasospasm. Healthcare providers should maintain a high index of suspicion in the appropriate clinical scenario for this life-threatening adverse effect of misoprostol so that timely and appropriate interventions can be delivered as the cardiovascular prognosis is favourable, as seen in our report.

\section{REFERENCES}

1. Tang OS, Gemzell-Danielson K, Ho PC. Misoprostol: pharmacokinetic profiles, effects on uterus and side-effects. Int J Gynecol Obstet 2007;99:160-167. doi: 10.1016/j. ijgo.2007.09.004

2. Prashanth A, Chakravarthy M, Maddirala P. Life threatening adverse reaction following prostaglandin analogue use in obstetric and gynecological practice. J Obstet Anesth Crit Care 2016;6:34-37. doi: 10.4103/2249-4472.181077

3. Illa M, Bennasar M, Eixarch E, Berge R, Font C, Palacio M. Acute coronary artery vasospasm associated with misoprostol for termination of pregnancy. Fetal Diagn Ther 2010;27:174-177. doi: 10.1159/000295923

4. . Owusu KA, Brennan J, Perelman A, Meoli E, Altshuler J. Nitroglycerin administration during cardiac arrest by coronary vasospasm secondary to misoprostol. J Cardiol Cases 2015;12(5):166-168. doi: 10.1016/j.jccase.2015.07.003.

5. Mazhar F, Sultana J, Akram S. Misoprostol-induced acute coronary syndrome in a premenopausal woman: a case report with literature review. Curr Drug Saf 2018;13(1):6568. doi: 10.2174/1574886312666171122100929

6. Sung CW, Jung JH, Lee SH, Lee KM, Ahn BM, Choi S, et al. Acute myocardial infarction due to vasospasm induced by prostaglandin. Can J Cardiol 2009;25(10):e359-360. doi: 10.1016/s0828-282x(09)70724-x

7. Qian YM, Jones RL, Chan KM, Stock AI, Ho JK. Potent contractile actions of prostanoid EP3-receptor agonists on human isolated pulmonary artery. Br J Pharmacol 1994;113(2):369-374. doi: 10.1111/j.1476-5381.1994.tb16997

8. Brennan A, Adams H, Galligan J, Whitbourn R. Coronary vasospasm and concurrent Takotsubo cardiomyopathy. Br J Cardiol 2019;26:38-50. doi: 10.5837/bjc.2019.011

9. Misumi I, Ebihara K, Akahoshi R, Hirota Y, Sakai A, Sanjo M, et al. Coronary spasm as a cause of Takotsubo cardiomyopathy and intraventricular obstruction. J Cardiol Cases 2010;2(2):e83-87. doi: 10.1016/j.jccase.2010.03.007 